\title{
Legibility Index for Examining Common Viewing Situations: A New Definition Using Solid Angle
}

\author{
Hongyi Cai, Ph.D. ${ }^{1}$, and Paul A. Green, Ph.D. ${ }^{2}$
}

Abstract-The legibility levels of text and graphics can be assessed using the Legibility Index, conventionally defined as the distance at which material can be read with perfect accuracy (the legibility distance) divided by the character height. The ratio equals the inverse tangent of the visual angle subtended by the character. The definition assumes that the viewing material is perpendicular to the observer, which is not always true. Although text and graphics are, in reality, often viewed not perpendicular to the display, they are rarely studied. To examine the legibility of characters viewed not perpendicular to the display, this paper has redefined the Legibility Index as the inverse square root of the solid angle subtended by the target, based on a hypothesis that the three-dimensional solid angle, rather than the twodimensional visual angle, both subtended by the character, captures how people recognize text and graphics that usually have two significant dimensions (width and height). This hypothesis proves consistent with how retinal images of text and graphics activate cones in the fovea of a viewer's eyes, and has also been verified in a laboratory using legibility data collected from 20 human subjects. Using these data, the calculated steady redefined Legibility Index closely matches the fact that the 20 subjects have similar recognition performance (critical legibility level with 100 percent accuracy) of text (same target) under the same lighting conditions $\left(L_{b}=120 \mathrm{~cd} / \mathrm{m}^{2}\right.$, $C_{\%}=97.9$, zero ambient light) at different viewing angles (perpendicular or not perpendicular to the displays), while the calculated ratio of legibility distance to character height rapidly decreases with increased viewing angles.

Keywords-Legibility Index, viewing angle, text and graphics, nonperpendicular viewing, solid angle, visual angle, equation.

1. (Corresponding author) Research Associate, Department of Building Services Engineering, Hong Kong Polytechnic University, Kowloon, Hong Kong, behcai@polyu.edu.hk

2. Research Professor, University of Michigan Transportation Research Institute, 2901 Baxter Rd., Ann Arbor, MI 48109, pagreen@umich.edu

(C) 2009 The Illuminating Engineering Society of North America

doi: 10.1582/LEUKOS.2008.05.04.002 


\section{INTRODUCTION}

P eading text and graphics is a common visual task. Viewing materials can be presented on a wide variety of displays such as computer screens, instrument panel displays, billboards, warning placards, signs, classroom projection screens, blackboards, maps, books, and magazines. These displays are often viewed not perpendicular to the observer. Their purposes are not well served, however, if the text and graphics are not legible or legible only when viewed perpendicularly but illegible from other viewing directions as desired in common viewing situations. Legibility of text or graphics refers to the observer's decipherability of the spatial mosaic of strokes of text or details of graphics presented at a specific scenario, to recognize the established forms of these characters and the embedded meanings thereof (Gove and others, 1986). How does the observer recognize text and graphics viewed at different angles? When viewed, text or graphics form a retinal image in the center fovea of a viewer's eyes. The shape and size of the retinal image varies with the viewing distance from the observer's eye to the characters and the viewing angle between the display normal and the observer's sight line. The retinal image then activates the underlying cones to fire signals to the cortex nerves in the brain. For text and graphics to be legible, their retinal images must be large enough for the underneath cones to discriminate, and must have enough lighting intensity and contrast to activate these cones. The observer's acuity level and age, viewing distance, viewing angle, and lighting condition are all important factors affecting the legibility of text and graphics.

\section{RESEARCH PROBLEM}

The legibility level of text and graphics is assessed in practice using a Legibility Index. The Legibility Index, as $L I$ in (1), has been conventionally defined as the ratio of the distance at which material can be read with perfect accuracy (the legibility distance) to the character height (United States Sign Council 1998). As illustrated in Fig. 1, the ratio equals the inverse tangent of the two-dimensional visual angle $V$ that text or graphics subtend to the viewer's eyes.

$L I=\frac{D}{H}=\frac{1}{\tan (V)}$

Where:

$L I=$ Conventional definition of the Legibility Index

$D=$ Legibility distance

$H=$ Normal height of the character

$V=$ Visual angle subtended by the height of character

The conventional definition assumes that the image being examined is perpendicular to the viewer, which is not always true. In common viewing situations, a viewer's sight line to text and graphics often deviates from the display normal. As illustrated in Fig. 2, when text or graphics are read not perpendicular to the display, their projected image, which is perpendicular to the sight line, rather than themselves, forms an inverted retinal image in the center fovea of the viewer's eyes. The size of this projected image is smaller than that of the normal character. The shape of this projected image also changes with the viewing angle. Accordingly, if extending the conventional definition $(L I=D / H)$ for notperpendicular-to-the-display viewing situations, the projected height, rather than the normal height, of the viewed text or graphics should be used to 


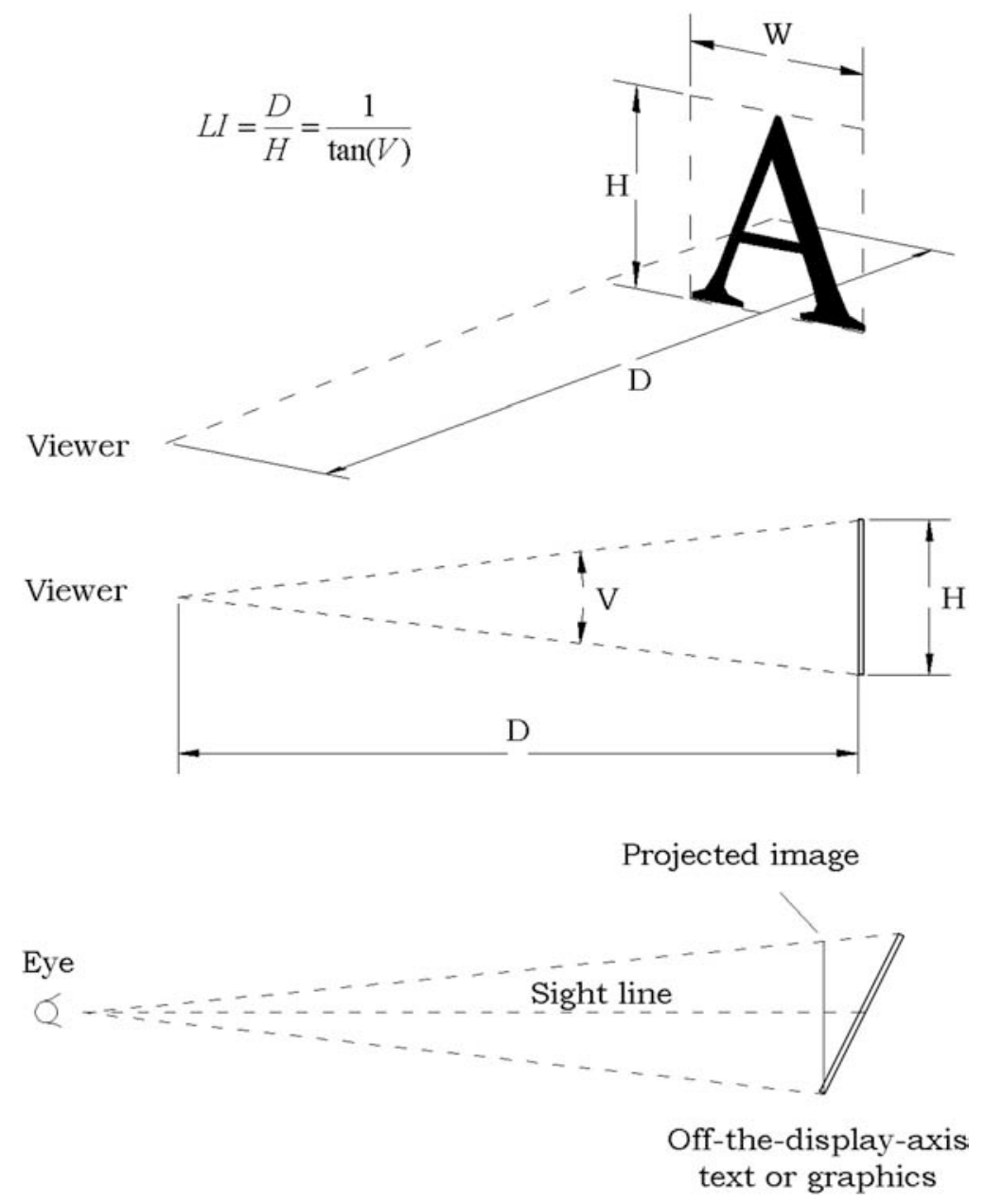

calculate the Legibility Index. Thus, to examine not-perpendicular-to-the-display viewing situations, why not simply replace the normal height with the projected height to redefine the Legibility Index? Unfortunately, this replacement works solely for linear targets, such as a line with no substantial width. For common nonlinear targets whose height and width are both substantial, width cannot be ignored, as illustrated in Fig. 3. In Fig. 3, letter A is viewed from four different points (P1, P2, P3, and P4), which are located on a horizontal plane at equal viewing distance $\mathrm{D}$ but at different viewing angles. The projected height of letter A remains constant $\mathrm{H}$ at these four points, while the projected width gradually decreases inversely with the viewing angles. Based on daily experience, at the same distance $\mathrm{D}$, letter $\mathrm{A}$ is more difficult to recognize when viewed off the display axis than when viewed perpendicularly.

\section{NEW DEFINITION OF LEGIBILITY INDEX}

To assess the legibility levels of characters viewed not perpendicularly to their displays, a new definition of the Legibility Index is considered necessary to replace the insufficient $D / H$ ratio. Such new definition should be rooted in an appropriate way to specify target size and might be indicated in previous research studies on visual performance. Shlaer (1937) proposed a discussion in
Fig. 1. Conventional definition of the Legibility Index (LI).

Fig. 2. Projected image of text or graphics viewed off the display axis. 
Fig. 3. Varying projected widths of letter $A$ viewed from P1, P2, P3, and P4 vs. constant projected height.

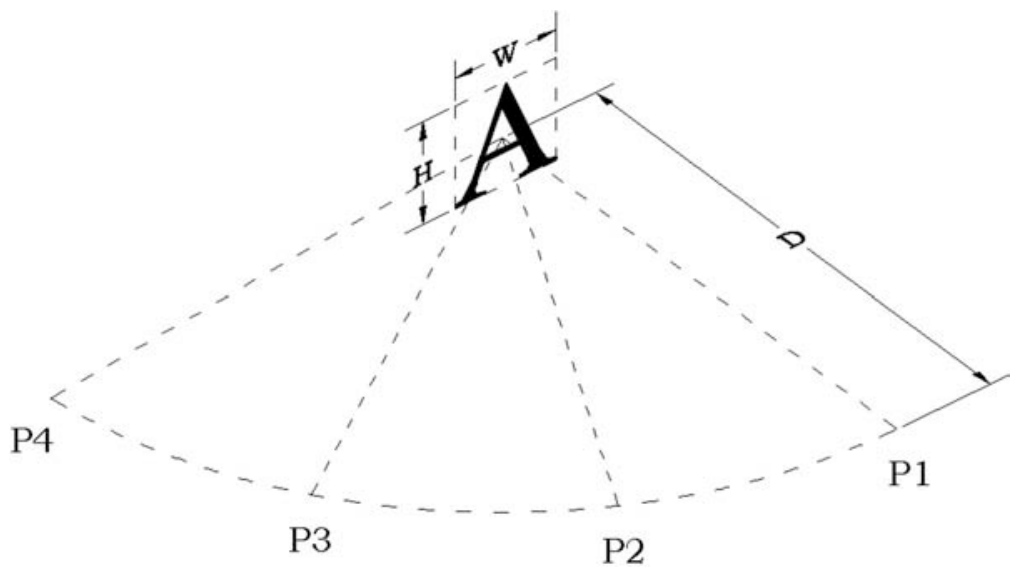

his study on detail perception that whether a visual area or a distance between bars of a grating is appropriate to specify the target size. He found that detail discrimination is a function of distance rather than visual area. Shlaer, Smith, and Chase (1942), Moon and Spencer (1944), Zwahlen and Schnell (1995), and Holick and Carlson (2002) developed equations for predicting visual angles subtended by legible Landolt or single-bar type targets, or text presented on traffic signs and license plate or roadway signs. Blackwell (1946) used circular stimuli varied in diameter from 3.6-121 arcminutes by projecting a spot of light on white screen for examining contrast thresholds of human eyes. Blackwell $(1959,1970,1980)$ and Blackwell and Blackwell $(1971,1980)$ used visual angles of 1-60 $(1,2,4,10,60)$ arcminutes to characterize the luminous discs or Landolt rings used in his studies for developing the visibility model formally adopted by CIE in 1981. Howell and Kraft (1960) also measured the legibility of alphanumeric symbols in four visual angles from 6 to 37 arcminutes. Bouma, Legein, Melotte, and Zabel (1982) measured oral reading rates for text whose sizes vary from $0.3^{\circ}$ to $2.8^{\circ}$. Legge, Pelli, Rubin, and Schleske (1985) examined reading rates for characters in sizes from 3.6' to $24^{\circ}$ at a range of 400:1. Bailey, Clear, and Berman (1993) used Bailey-Lovie word reading charts on which the strokewidth $(1 / 5$ of the height) subtends $0.6-6$ arcminutes to measure subjects' reading speed. All these studies used visual angle to specify the target size, which supports the conventional definition of Legibility Index. However, noticing the limitations of visual angle for specifying target size in a linear way solely, other researchers specified target size in two dimensions, both width and height. For example, Campbell and Robson (1968) used $2^{\circ} \times 2^{\circ}$ at viewing distance of $285 \mathrm{~cm}$ and $10^{\circ} \times 10^{\circ}$ at $57 \mathrm{~cm}$ to specify a $10 \times 10 \mathrm{~cm}$ aperture in which sine-, square-, saw-tooth-, and rectangular-wave gratings were presented as materials for examining contrast sensitivity vs. spatial frequency. Rea (1986) defined the middle digits in the numerical verification task experiment as of 13 arcminutes wide and 19 arcminutes high. Rea and Quellette (1988) also specified the visual field as of $12^{\circ}$ wide and $7^{\circ}$ high where targets were generated on a video screen in the reaction time experiment. It was further realized by researchers that visual area of a target subtending solid angles at different distances is actually a more appropriate way for characterizing target size than visual angle. Kristofferson (1957) found in his experiment that targets of different shapes (square, discs, triangles, etc.) have almost equivalent threshold visibility as long as they subtend the same solid angle (Dember 1965; Rea and Quellette 1991). Rea and Quellette $(1988,1991)$ used solid angles from $2 \times 10^{-6}$ to $2.8 \times 10^{-3}$ steradians 
to specify the target size in their reaction time experiment reading square targets flashed on video screen for developing the relative visual performance (RVP) model. Similar ways for specifying target size were also used in the studies of Kristofferson (1957), Campbell and Robson (1968), Rubin and Siegel (1984), Legge and others, (1985), and Rea and Kambich (Rea and Quellette 1991). Rea and Quellette (1991) proposed a hypothesis that visual area of a target subtending in solid angle is better than width, height, or detail spacing, which subtends in visual angle, to specify target size for both threshold and suprathreshold visual performance, but without further experimental verification, goal of present study.

Indicated from those previous studies, this paper has developed the new definition of Legibility Index as the inverse square root of solid angle $\omega$ subtended by the viewing target, as $L I^{\prime}$ in (2), based on the hypothesis below. The choice of the inverse square root of solid angle $\omega$ rather than simple reciprocal of $\omega$ for the form of the new definition is based on two reasons. First, different from threshold detection for visibility, legibility deals with suprathreshold visual performance, where Piper's law $(L \times \sqrt{A}=C$, where $\mathrm{L}=$ threshold luminance of the stimulus, $\mathrm{A}=$ area of the stimulus, $\mathrm{C}=$ constant) holds for a legible character slightly larger than the critical area (Boff, Kaufman, and Thomas 1986). In Piper's law, target area $A$ is in square root that shall be compatible with the new definition. Second, the new definition should also be consistent in dimension with the conventional definition for perpendicular viewing.

$L I^{\prime}=\sqrt{\frac{1}{\omega}}$

Where:

$L I^{\prime}=$ Redefined Legibility Index for both perpendicular and nonperpendicular viewing

$\omega=$ Solid angle subtended by any text and graphics to the retina of the observer's eyes

Hypothesis: the three-dimensional solid angle $\omega$, rather than the twodimensional visual angle $V$, both subtended by the characters under viewing, captures how people recognize text and graphics that usually have two significant dimensions (width and height).

$L I^{\prime}$ is the essential form for assessing the legibility levels of text and graphics viewed both perpendicularly and not perpendicularly. $L I^{\prime}$ can be further developed into (4) by substituting the definition of solid angle $\omega$, as defined in (3) and illustrated in Fig. 4. In addition, based on the geometrical relationship shown in Fig. 5, the incident angle $\xi$ in (4) can be substituted by the horizontal viewing angles $\phi$ and the vertical viewing angle $\alpha$, using $\cos \xi=\cos \phi \cos \alpha$, we get (5). Equation (5) is the expanded form of the redefined Legibility Index $\left(L I^{\prime}\right)$ for common not-perpendicular-to-the-display viewing situations, taking into account horizontal and vertical viewing angles. In particular, (5) also examines perpendicular viewing situations when zero value is assigned to the horizontal and vertical viewing angles. Thus, the redefined Legibility Index $L I^{\prime}$ in (5) fits general viewing situations.

$\omega=\frac{A}{D^{2}} \cos \xi$ 
Fig. 4. Definition of solid angle $\omega$ (Rea 2000, Fig. 9-1, p. 9-2).
Fig. 5. Off-the-display-axis viewing of target and the incident angle $\xi$.
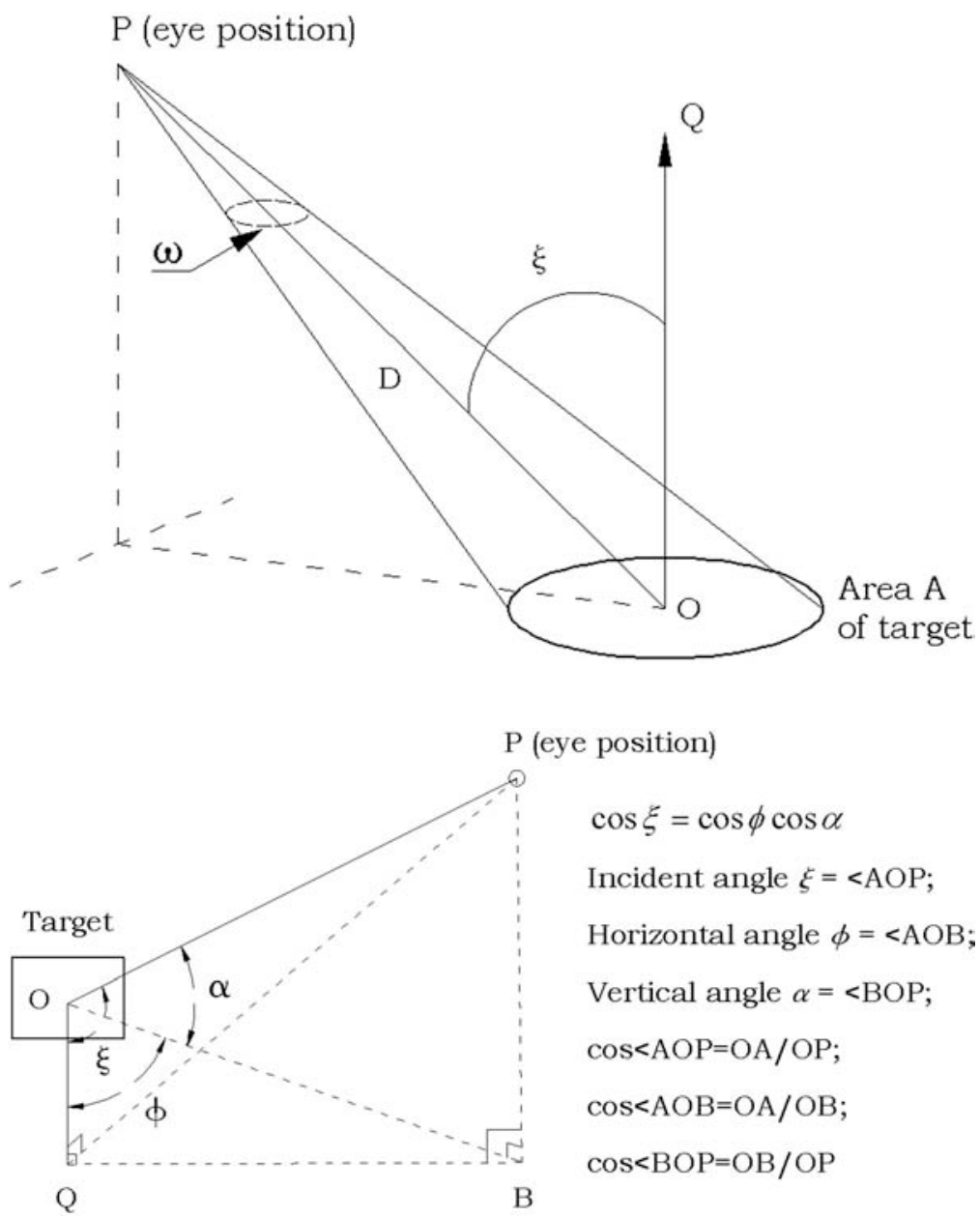

Where:

$\omega=$ Solid angle subtended by character to the retina of observer's eyes

$\xi=$ Incident angle between the sight line OP and display normal OQ

$A=$ Normal character area

$D=$ Viewing distance

$$
\begin{aligned}
& L I^{\prime}=\frac{D}{\sqrt{A \cdot \cos \xi}}=\frac{D}{\sqrt{A}}(\cos \xi)^{-0.5} \\
& L I^{\prime}=\frac{D}{\sqrt{A \cdot \cos \phi \cos \alpha}}=\frac{D}{\sqrt{A}}(\cos \phi)^{-0.5}(\cos \alpha)^{-0.5}
\end{aligned}
$$

Where:

$L I^{\prime}=$ Redefined Legibility Index for both perpendicular and nonperpendicular viewing

$D=$ Legibility distance

$A=$ Normal character area

$\xi=$ Incident angle between the sight line OP and display normal OQ

$\phi=$ Horizontal viewing angle

$\alpha=$ Vertical viewing angle 
The normal area $(A)$ examined in (4) and (5) is measured differently for letters or graphics. Letters $A \sim Z$ have different established forms. The spatial distribution of strokes, including strokewidth, orientations of strokes, and spacing between strokes, determines which letter it is. Even for the same letter, the spatial distribution of its strokes varies with font. Therefore, the spatial mosaic of each letter's strokes is crucial for its legibility, as important as the size of the letter. This finding is also true for some specific graphics with established forms (for example, $\geqslant, /, \infty, \times, \div)$. Conclusively, the normal area $(A)$ of letters or specific graphics with established forms should be defined as the product of width multiplied by height, to take both the orientations of text strokes or graphic details and the spacing between them into account, as shown in Fig. $6 \mathrm{a}$ and 6b.

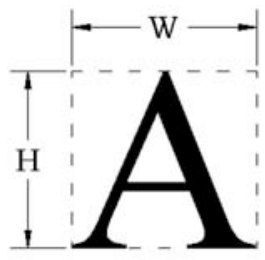

$\mathrm{A}=\mathrm{W} \times \mathrm{H}$

(a) letters

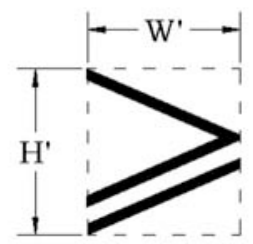

$\mathrm{A}=\mathrm{W}^{\prime} \mathrm{xH}$

(b) graphics with established forms

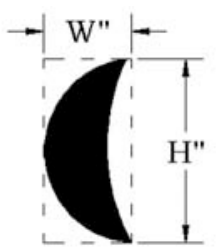

$A=$ shaded area, not W"xH"

(c) random graphics
Fig. 6. Different measurements of normal area $A$ : text $(A=W \times H)$; Graphics with established forms $\left(A=W^{\prime} \times H^{\prime}\right)$, and Random graphics $(A=$ area summation of all details).

In practice, areas of text have been measured using height and width. However, for random graphics, the spatial mosaic of the details has no established forms and preset meaning for their legibility. The normal area $(A)$ of random graphics is therefore defined as the summation of the areas of all details, as shown in Fig. 6c.

For text and graphics using width and height to define their normal area $A$ ( $A$ $=W \cdot H$ ), (5) can be further expounded as (6) by replacing A with width $W$ and height $H$. When $\phi=0, \alpha=0$ in (6), we get (7) to calculate the Legibility Index $L I^{\prime}$ perpendicular for perpendicular viewing. Comparing (7) and (1), we get (8). Apparently, the conventional definition of Legibility Index assumes that the height-to-width ratio of any text or graphics equals 1 , which is not always true in reality. By using (8), values of the conventional Legibility Index $L I$, which are widely accepted in the signage industry, can be converted into the redefined definition $L I^{\prime}$ perpendicular.

$\left.L I^{\prime}=\frac{D}{\sqrt{A \cdot \cos \phi \cos \alpha}}=\frac{D \cdot \sqrt{r}}{H}(\cos \phi)^{-0.5}\right)(\cos \alpha)^{-0.5}$

Where:

$L I^{\prime}=$ Redefined Legibility Index for both perpendicular and nonperpendicular viewing

$D=$ Legibility distance

$A=$ Normal character area,

$A=W \cdot H$, for text and graphics using width and height to define area

$H=$ Character height

$W=$ Character width

$r=$ Height-to-width ratio of character, $r=H / W$

$\phi=$ Horizontal viewing angle

$\alpha=$ Vertical viewing angle 


$$
\begin{aligned}
& L I_{\text {perpendicular }}^{\prime}=\frac{D}{\sqrt{A}}=\frac{D}{H} \cdot \sqrt{r} \\
& L I_{\text {perpendicular }}^{\prime}=L I \cdot \sqrt{r}
\end{aligned}
$$

Where:

$L I_{\text {perpendicular }}^{\prime}=$ Redefined Legibility Index for perpendicular viewing only

$D=$ Legibility distance

$A=$ Normal character area, $A=W \cdot H$, for characters using width and height to define area

$H=$ Character height

$W=$ Character width

$r=$ Height-to-width ratio of character, $r=H / W$

$L I=$ Conventional definition of Legibility Index

\section{VERIFICATION OF THE HYPOTHESIS}

\subsection{PHYSIOLOGICAL FOUNDATRION}

For legibility, the retinal images of materials are focused in the center fovea of an observer's eyes which, given enough light, activate the underlying cones. These foveal cones are very tightly packed and form a two-dimensional triangular array, with no strong orientation dependencies, as illustrated in Fig. 7 (Wandell 1995). This dense representation of the foveal cones suggests that the spatial mosaic of the cones must be important for the visual encoding (recognition) of text and graphics (Wolken 1966; Hendee 1993; Wandell 1995; Boff and others, 1986). The legibility level of characters viewed perpendicularly to their displays or not is eventually determined by the quantity and spatial distribution of the activated cones. Different text or graphics have different spatial distributions of their activated cones in the center fovea. The conventional definition of Legibility Index $(L I=D / H)$ uses only one dimension (usually height) of the characters to assess their legibility level; width is irrelevant. The reasonable explanation for this assumption is that the cones activated by the retinal images must be linearly distributed in the center fovea of the viewer's eyes. In reality, this is true only for a line or other linear targets with no width, as illustrated in Fig. 7a. For linear targets, visual angle $V(V=\arctan (H / D)$, as shown in Fig. 1 previously) is sufficient for assessing their legibility. However, an absolute linear viewing target is uncommon. For a wide variety of nonlinear targets in reality, the retinal images usually have two significant dimensions (both width and height) and activate a two-dimensional collection of cones, as illustrated in Fig. 7b. For nonlinear targets, the two-dimensional visual angle $V$ is insufficient for examining the two-dimensional collection of activated cones. Instead, the threedimensional solid angle $\omega$ should be used for this purpose.

\subsection{LABORATORY EXPERIMENT}

To further verify the hypothesis, an experiment was carried out in a lighting laboratory at the University of Michigan Transportation Research Institute (UMTRI). In this experiment, 20 young human subjects were asked to recognize viewing materials presented at 16 different incident angles under the same lighting condition (image background luminance $\mathrm{L}_{\mathrm{b}}=120 \mathrm{~cd} / \mathrm{m}^{2}$, image contrast $\mathrm{C}_{\%}=97.9$, zero ambient light). Fig. 8 shows the experimental setup under full 

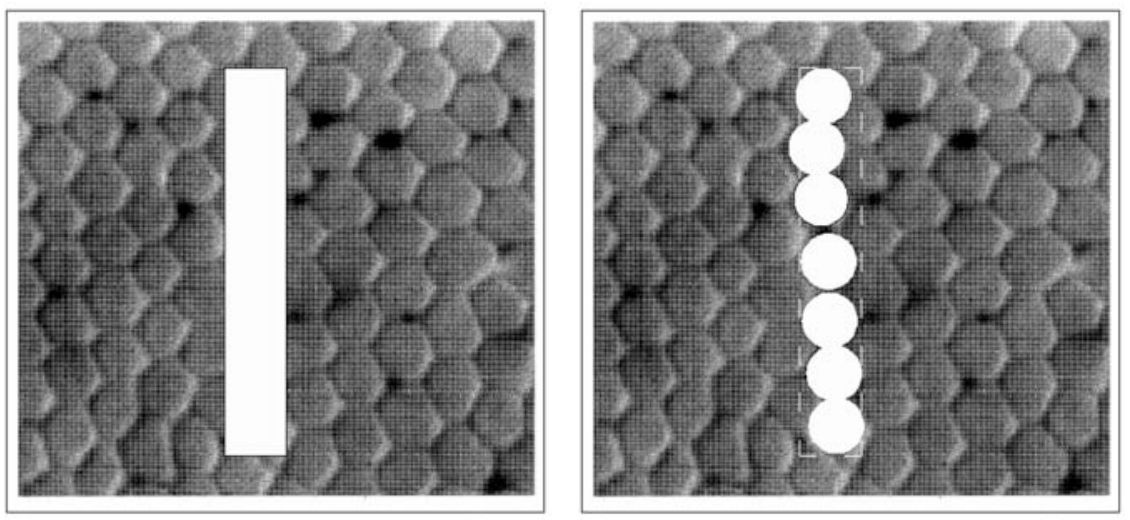

(a) Linearly distributed cones activated to recognize a bar
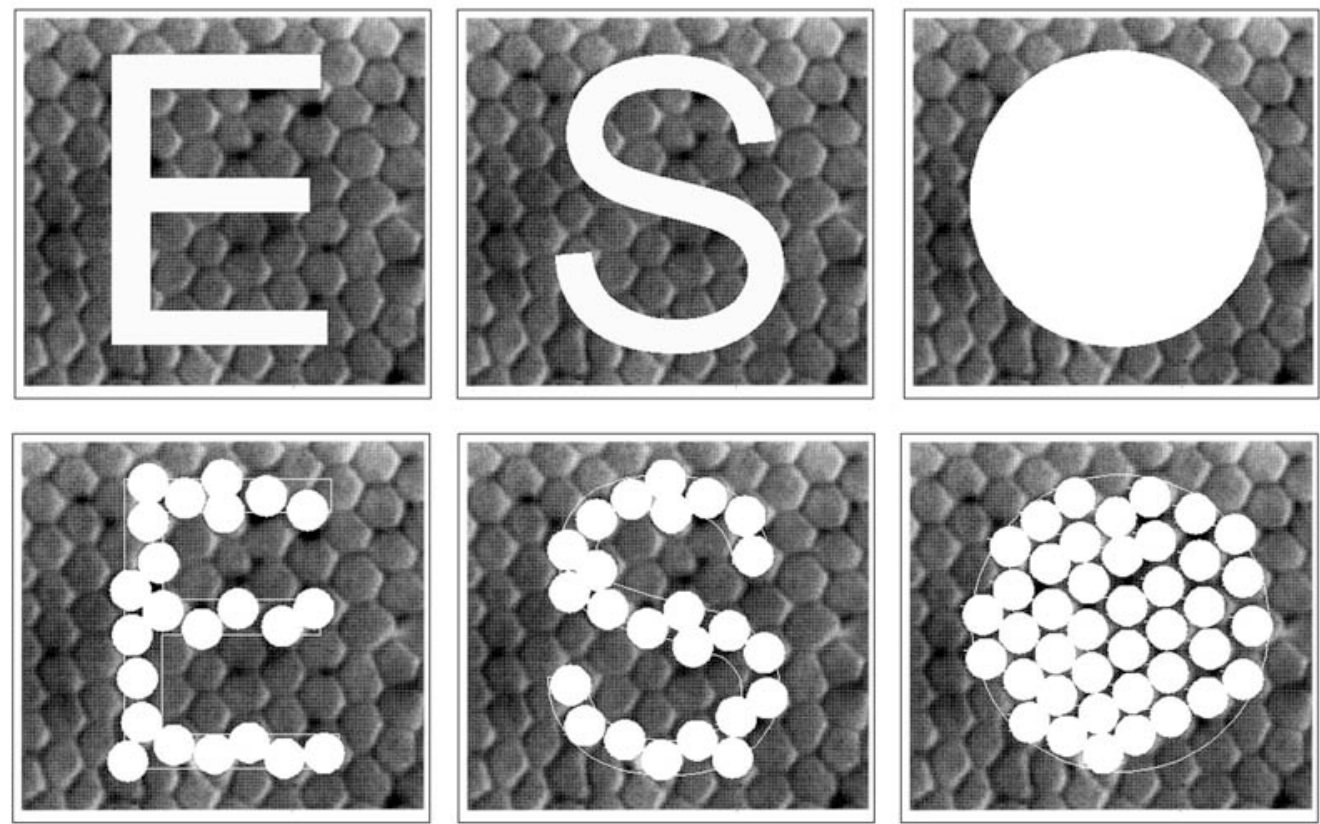

(b) Two-dimensional distribution of the activated cones

ceiling light, which was turned off during the experiment to maintain a zero ambient light level.

As shown in Fig. 8, a dimmable light case lit by two fluorescent T8 tubes (daylight white) was placed on a desk at one end of the laboratory while subjects

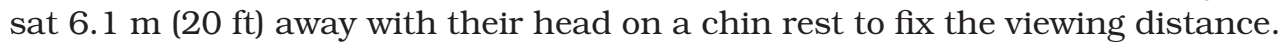
Subjects were required to be 20-29 years old (to minimize the aging effect of human eyes on legibility), with binocular eyesight of $20 / 20$ or better $(20 / 20$, $20 / 16$, or $20 / 12.5$, with or without glasses) and normal color vision. Visual materials were attached to the opalescent surface of the light case, whose surface luminance $\left(\mathrm{L}_{\mathrm{b}}\right)$ was able to be dimmed from $1150 \mathrm{~cd} / \mathrm{m}^{2}$ to $16 \mathrm{~cd} / \mathrm{m}^{2}$, with an average uniformity of 89.5 percent $(\min / \max )$ at 5 different light levels $\left(22.2,63.5,117.0,485.7\right.$, and $\left.1150 \mathrm{~cd} / \mathrm{m}^{2}\right)$. During the experiment, the light case was horizontally rotated $\left(\phi=0^{\circ}, 30^{\circ}, 60^{\circ}, 75^{\circ}\right)$ and vertically tilted $\left(\alpha=0^{\circ}\right.$,
Fig. 7. Retinal images of a bar, text $(E, S)$ and a disc in the fovea with underlying activated cones (bright spots), the background pixels (dark spots) are the spatial mosaic of cones in the fovea (Wandell 1995, Fig. 3.4, p. 49). 
Fig. 8. Equipments installed in the lighting laboratory at UMTRI.

TABLE 1.

Total 16 incident angles $\xi$ examined in the laboratory experiment $(\cos \xi=\cos \phi \cos \alpha)$
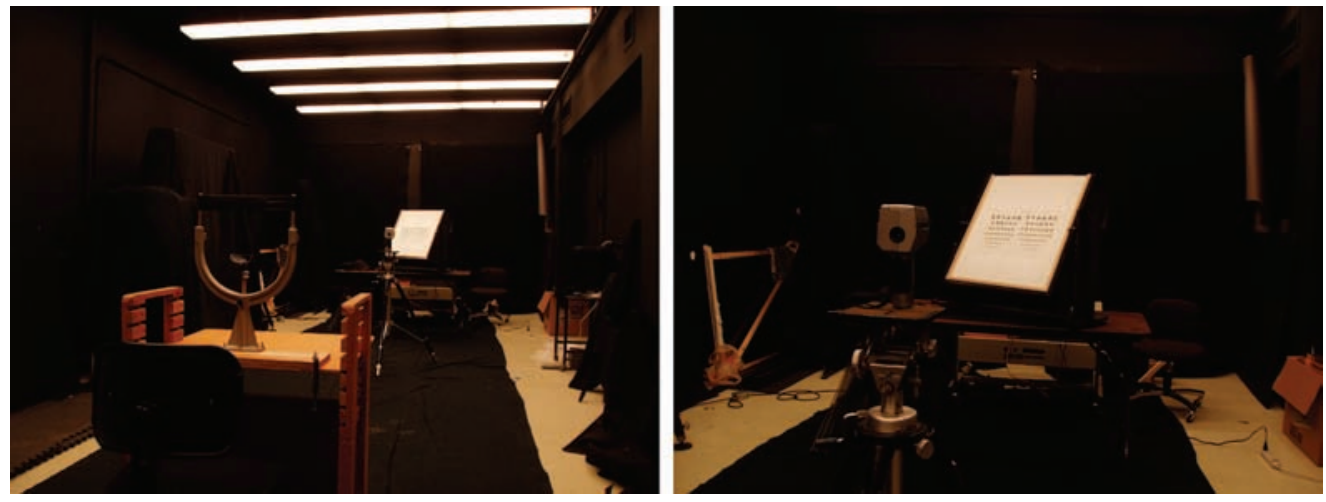

\begin{tabular}{|c|c|c|}
\hline $\begin{array}{c}\text { Horz. angle } \phi \\
\text { in deg }\end{array}$ & $\begin{array}{c}\text { Vert. angle } \alpha \\
\text { in deg }\end{array}$ & $\begin{array}{c}\text { Incident angle } \xi \\
\text { in deg }\end{array}$ \\
\hline 0 & 0 & 0.0 \\
\hline 30 & 0 & 30.0 \\
\hline 0 & 31.5 & 31.5 \\
\hline 30 & 31.5 & 42.4 \\
\hline 0 & 46.5 & 46.5 \\
\hline 30 & 46.5 & 53.4 \\
\hline 60 & 0 & 60.0 \\
\hline 0 & 61 & 61.0 \\
\hline 60 & 31.5 & 64.8 \\
\hline 30 & 61 & 65.2 \\
\hline 60 & 46.5 & 69.9 \\
\hline 75 & 0 & 75.0 \\
\hline 60 & 61 & 76.0 \\
\hline 75 & 31.5 & 77.3 \\
\hline 75 & 46.5 & 79.7 \\
\hline 75 & 61 & 82.8 \\
\hline
\end{tabular}

$31.5^{\circ}, 46.5^{\circ}, 61^{\circ}$ ) to provide $4 \times 4=16$ incident angles $\xi$, as calculated in TABLE 1. To simulate an ideal diffusive surface, the surface luminance $\left(\mathrm{L}_{\mathrm{b}}\right)$ of the light case was purposely dimmed to a constant $120 \mathrm{~cd} / \mathrm{m}^{2}$ for all 16 viewing angles. A recently calibrated meter Minolta LS-100 mounted on a tripod was used to measure the luminances in this experiment.

In terms of visual materials, this experiment used letter Es as a single visual task to test the hypothesis because of two reasons. First, legibility is a task performance involving nonvisual components such as eye motions and brain cognition (Kambich 1991). Materials presented for legibility have sufficient exposure time, which is much longer than the detection time quantified in Rea's Reaction Time (RT) model (Clear and Berman 1990). Findings of Kristofferson (1957), Campbell and Robson (1968), Legge and others, (1985), Rubin and Siegel (1984), Rea and Kambich (cited in Rea and Quellette 1991) and Rea and Quellette (1991) that target details or high spatial frequencies are relatively unimportant for visual detection (visibility) when compared to the visual area is not justified for legibility tasks (Bailey, Clear, and Berman 1993; Rea and Quellette 1991). Clearly, the spatial mosaic of strokes of text or details of graphics is critical for legibility. Legibility levels of different characters subtending equivalent solid angle vary with their high spatial frequency. Consequently, to exclude individual distraction on legibility, a single character is preferred than 


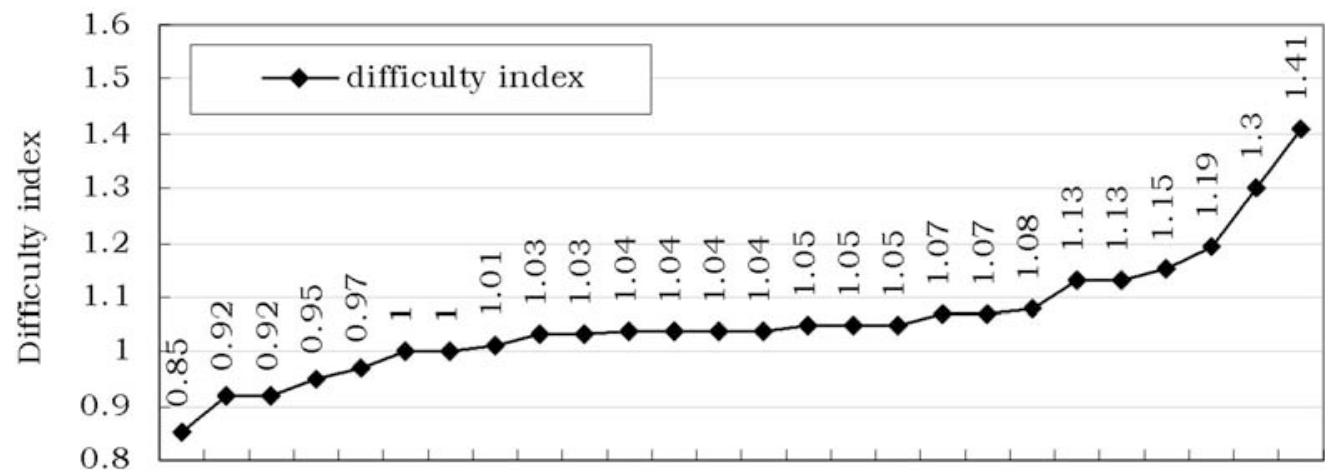

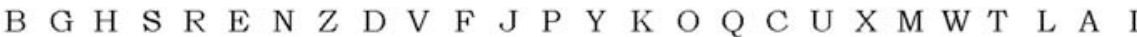
Easier to read Letter A-Z
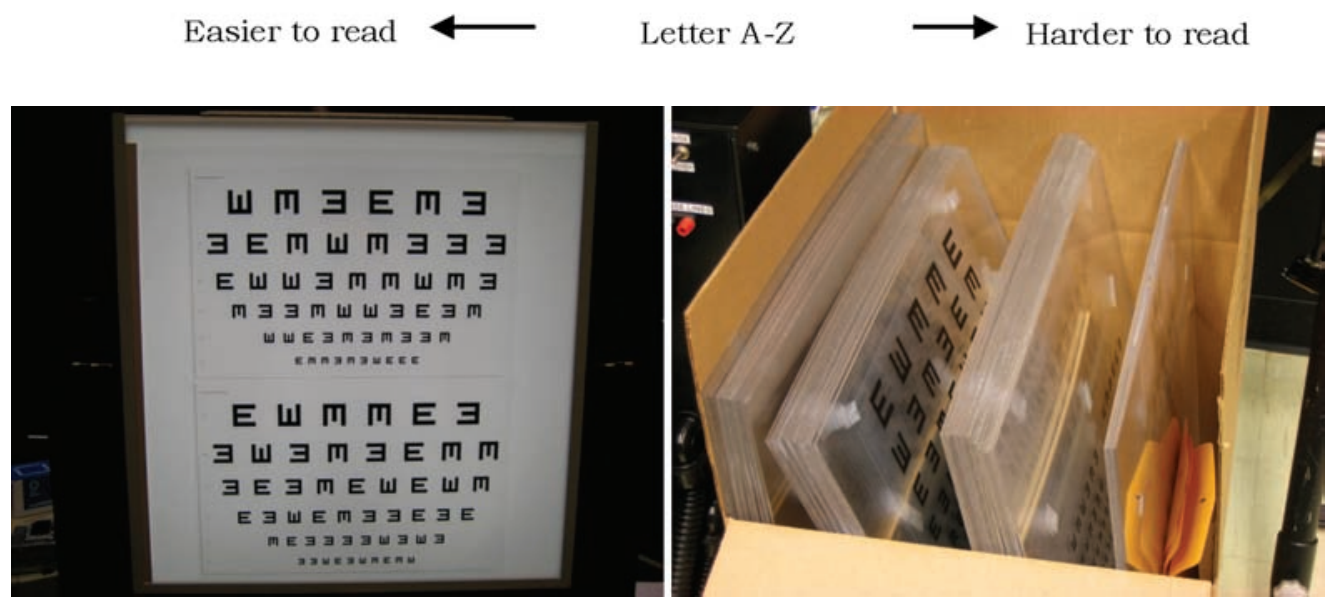

simplify the experiment and concentrate on the key variables (solid angle $\omega$ and visual angle $V$ subtended by the viewing target at different incident angle $\xi$ ) to verify the hypothesis. Second, legibility tasks in daily life, like reading signs, commonly involve text as whole or partial target, thus, a letter is used in the experiment whose high spatial frequency information is representative of all types of single character for testing the hypothesis purpose. The readability of 26 letters with different high spatial frequency is shown in Fig. 9. The eventual choice is the letter $\mathrm{E}$ with a height-to-width ratio $\mathrm{H} / \mathrm{W}=1$ and a height-tostrokewidth ratio $\mathrm{H} / \mathrm{Sw}=5$, because of its easy readability (difficulty index equals 1 in Fig. 9) and popularity in practice. As shown in Fig. 10, seven lines of letter Es of increasing size, random orientation, and positive contrast of black on white with average luminance contrast of $97.9\left(\mathrm{C}_{\%}\right)$ constitute a single E-chart, which was printed on transparencies and then attached on clear acrylic sheets. Subjects were asked to recognize the orientations of different lines of letter Es. Usually, two E-charts of the same sizes of letter Es but different random orientations were used together and attached on one clear acrylic sheet, to double check the reading performance of subjects at threshold legibility with 100 percent accuracy. A total of $48 \mathrm{E}$-chart sheets were used in this experiment to accommodate three levels of eyesight $(20 / 20,20 / 16,20 / 12.5)$ and 16 incident angles.

Each subject had 16 tests during his/her participation, corresponding to the 16 incident angles $(\xi)$ listed in TABLE 1 previously. Fig. 11 shows the actual
Fig. 9. Index of legibility difficulty for standard highway alphabet letters A-Z (Zwahlen and Schnell 1999, Fig. 2, p. 144).

Fig. 10. E-charts as viewing materials. 
Fig. 11. Viewing scenarios of 16 tests in the laboratory for acuity 20/20 with constant background luminance, image contrast, and zero ambient light.
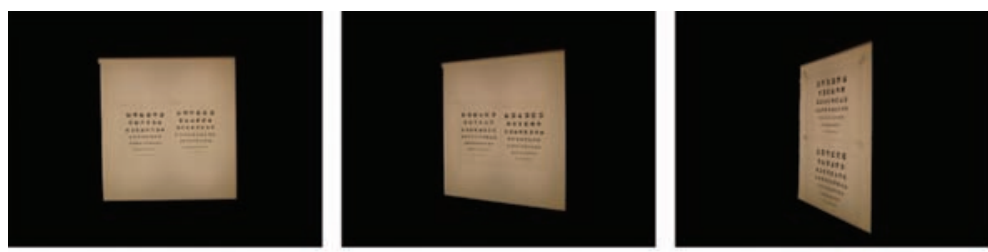

$\alpha=0^{\circ}, \phi=0^{\circ}$

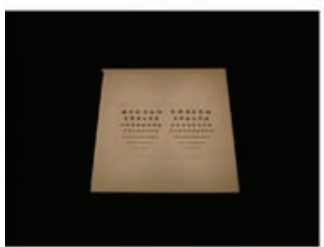

$\alpha=31.5^{\circ}, \phi=0^{\circ}$

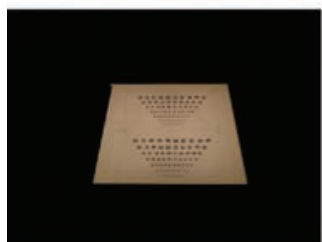

$\alpha=46.5^{\circ}, \phi=0^{\circ}$

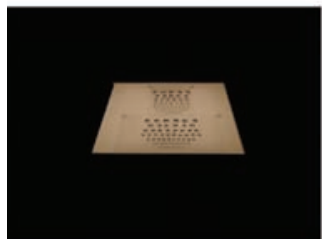

$\alpha=61^{\circ}, \phi=0^{\circ}$ $\alpha=0^{\circ}, \phi=30^{\circ}$

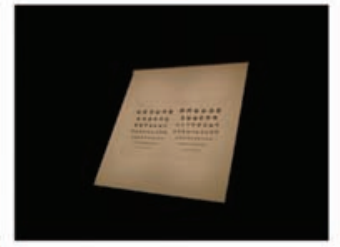

$\alpha=31.5^{\circ}, \phi=30^{\circ}$

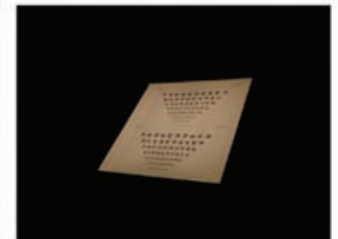

$\alpha=46.5^{\circ}, \phi=30^{\circ}$

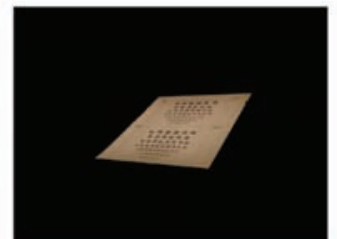

$\alpha=61^{\circ}, \phi=30^{\circ}$ $\alpha=0^{\circ}, \phi=60^{\circ}$

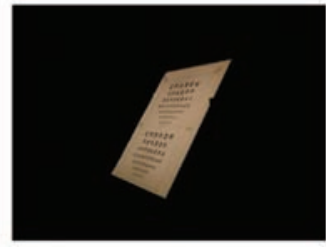

$\alpha=31.5^{\circ}, \phi=60^{\circ}$

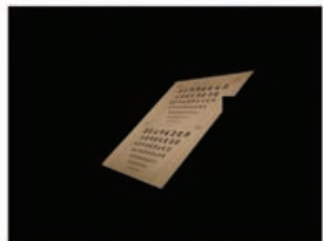

$\alpha=46.5^{\circ}, \phi=60^{\circ}$

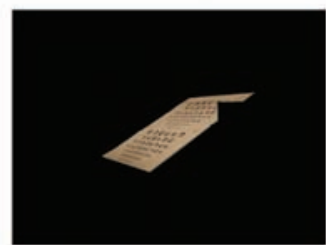

$\alpha=61^{\circ}, \phi=60^{\circ}$

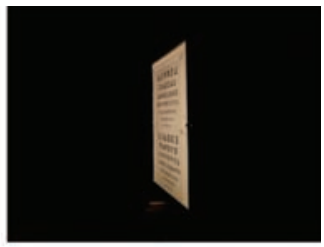

$\alpha=0^{\circ}, \phi=75^{\circ}$

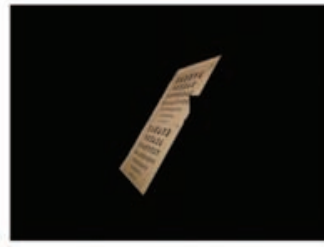

$\alpha=31.5^{\circ}, \phi=75^{\circ}$

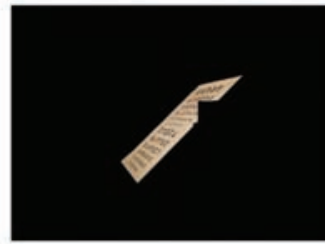

$\alpha=46.5^{\circ}, \phi=75^{\circ}$

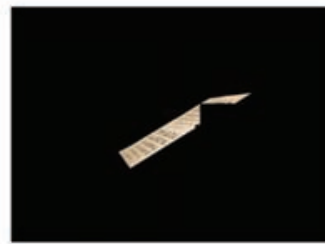

$\alpha=61^{\circ}, \phi=75^{\circ}$

viewing scenarios of the 16 tests for acuity level 20/20 in the laboratory. For each test, background luminance of letter $\mathrm{Es} \mathrm{L}_{\mathrm{b}}=120 \mathrm{~cd} / \mathrm{m}^{2}$, image luminance contrast $\mathrm{C}_{\%}=97.9$, and ambient light remained zero. The constant lighting condition excluded the noise of lighting variation on legibility, and thus focused on the viewing angles to compare the conventional and redefined Legibility Index at on- and off-axis viewing situations. The threshold legible heights $(H)$ of text recognized by the 20 subjects with 100 percent accuracy (no guessing was allowed) at each of the 16 tests were collected and then used to calculate the redefined Legibility Index, as $L I^{\prime}$ in (6) previously, and the $D / H$ ratio $(D$ is the legibility distance at constant $6.1 \mathrm{~m}$ ). In the perpendicular-to-the-display viewing, the $D / H$ ratio is the conventional Legibility Index, as $L I$ in (1) previously. In the other 15 nonperpendicular tests, the $\mathrm{D} / \mathrm{H}$ ratio no longer represents $L I$, but was used only for comparison to verify the hypothesis. The calculated $L I^{\prime}$ and $\mathrm{D} / \mathrm{H}$ ratio at three acuity levels and 16 incident angles are illustrated in Fig. 12 .

Three outcomes are illustrated in Fig. 12. First, for each eyesight level (20/20, $20 / 16$, or 20/12.5), except for perpendicular viewing, gaps exist between the values of $L I^{\prime}$ and $D / H$ ratio at the 15 not-perpendicular-to-the-display viewing situations, and rapidly increase with the incident angles. Second, when incident angle $\xi$ is less than approximately 708, subjects with the same eyesight level have steady reading performance, assessed by the almost equivalent $L I^{\prime}$. When 


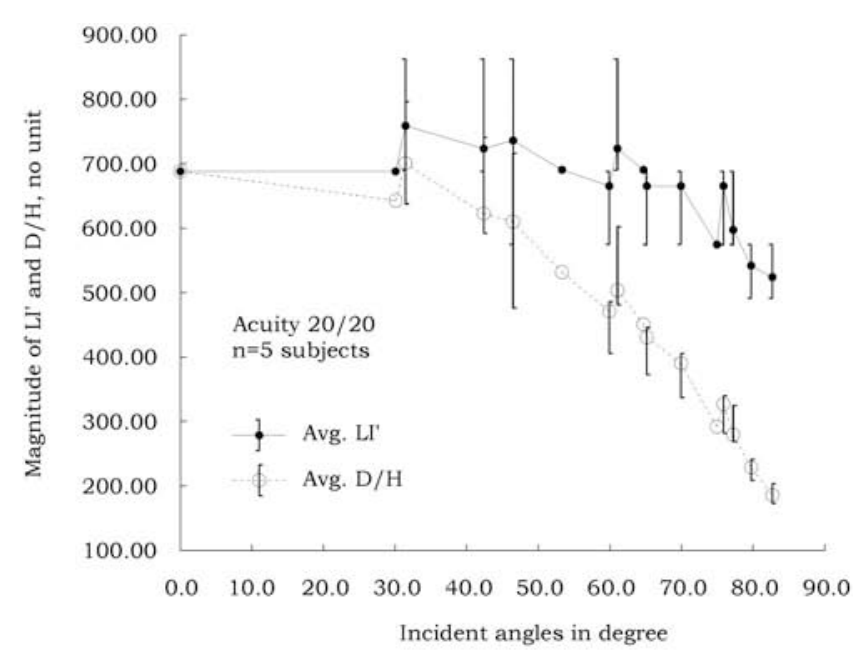

(a) $\mathrm{LI}^{\prime}$ vs. $\mathrm{D} / \mathrm{H}$ for 5 subjects with $20 / 20$ acuity

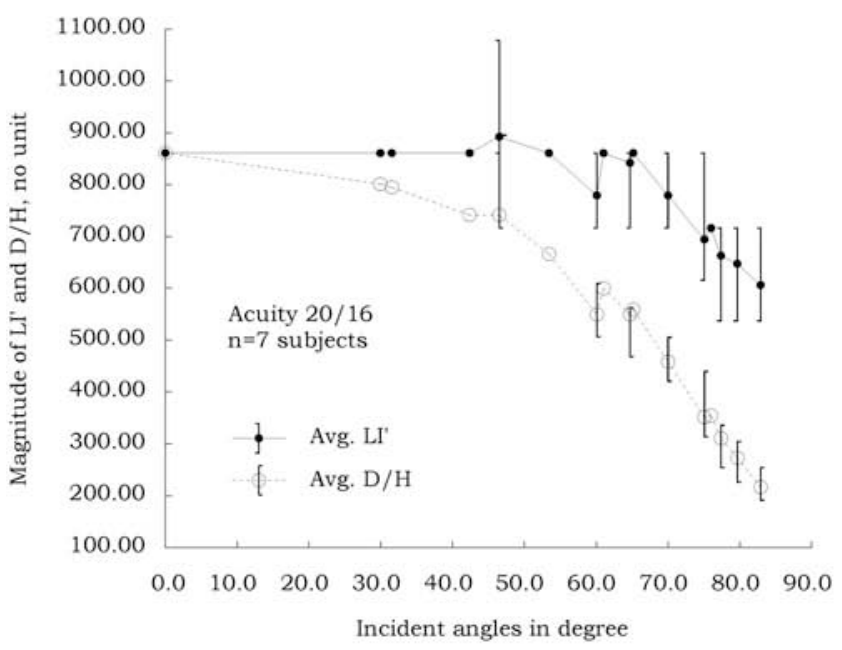

(b) $\mathrm{LI}^{\prime}$ vs. $\mathrm{D} / \mathrm{H}$ for 7 subjects with $20 / 16$ acuity

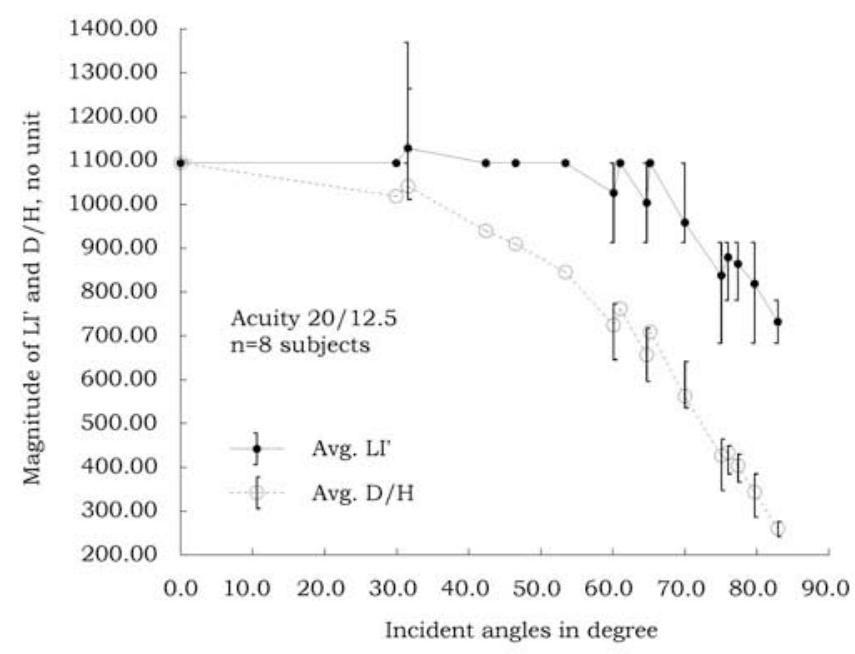

(c) LI' vs. D/H for 8 subjects with $20 / 12.5$ acuity
Fig. 12. Redefined Legibility Index $\left(L I^{\prime}\right)$ vs. $\mathrm{D} / \mathrm{H}$ ratio of legibility distance to the character height at 16 viewing angles. 
the incident angle $\xi$ is larger than approximately $70^{\circ}$, subjects' reading performance decreases, as indicated by the decreased $L I^{\prime}$. Third, in contrast to the steady values of $L I^{\prime}$ at incident angles from $0^{\circ}$ to $70^{\circ}$, the $D / H$ ratios keep going down when the viewing angle is increased. Theoretically, the power for discriminating characters is determined by the viewer's eyesight, the size and geometrical characteristics of text or graphic viewed, lighting conditions (for example, target luminance $\mathrm{L}_{t}$, background luminance $\mathrm{L}_{\mathrm{b}}$, adapting luminance $\mathrm{L}_{\mathrm{a}}$, and luminance contrast C), color difference, lamp spectrum, and other physical factors, but is independent of the viewing angles for looking at the target. In Fig. 12 , when incident angle $0^{\circ} \leq \xi \leq 70^{\circ}$, the steady redefined Legibility Index $L I^{\prime}$ closely matches the fact that the 20 subjects have the same recognition performance (threshold legibility levels with 100 percent accuracy) of letter Es under the same lighting conditions $\left(\mathrm{L}_{\mathrm{b}}=120 \mathrm{~cd} / \mathrm{m}^{2}, \mathrm{C}_{\%}=97.9\right.$, zero ambient light). However, when the target is viewed extremely off axis $\left(\xi>70^{\circ}\right)$, their retinal images are severely distorted, which harms the observer's reading habit and starts to dominate the reading performance and decrease the $L I^{\prime}$. As shown in Fig. 12, the enlarging gaps between the steady $L I^{\prime}$ and decreasing $\mathrm{D} / \mathrm{H}$ ratio at increasing viewing angles indicate that the $D / H$ ratio is no longer appropriate for assessing the legibility levels of text viewed not perpendicular to the display. Consequently, the redefined Legibility Index $\left(L I^{\prime}\right)$ better reflects the discrimination power of human eyes in a specific visual environment, and is thus validated for replacing the $D / H$ ratios for common viewing.

\section{APPLICATION OF THE NEW DEFINITION IN PRACTICE}

The redefined Legibility Index $\left(L I^{\prime}\right)$ has two different applications in practice. First, $L I^{\prime}$ can be used to assess the legibility levels of text or graphics viewed not perpendicularly across the viewing scenario, calculated using (4), (5) or (6) previously. Second, when the legibility levels of text or graphics are already known or assigned for a specific viewing scenario, (9) or (10), which are re-expressed from (6), can then be used to predict the legible size and orientation of characters viewed across the scene. Specifically, (10) is used for random graphics whose area cannot be simply calculated using width and height.

$$
\begin{aligned}
& H=\frac{D \cdot \sqrt{r}}{L I^{\prime}}(\cos \phi)^{-0.5}(\cos \alpha)^{-0.5} \\
& A=\frac{D^{2}}{\left(L I^{\prime}\right)^{2} \cdot \cos \phi \cos \alpha}
\end{aligned}
$$

Where:

$$
\begin{aligned}
L I^{\prime}= & \text { Redefined Legibility Index for both perpendicular and nonperpendicular } \\
& \text { viewing } \\
D= & \text { Legibility distance } \\
H= & \text { Character height } \\
W= & \text { Character width } \\
A= & \text { Normal character area } \\
A & =W \cdot H, \text { for characters using width and height to define area } \\
A & =\text { summation of areas of all details, for random graphics } \\
r & =\text { Height-to-width ratio of character, } r=H / W \\
\phi & =\text { Horizontal viewing angle } \\
\alpha & =\text { Vertical viewing angle }
\end{aligned}
$$


Both conventional and new definitions of Legibility Index have no unit. For convenience, however, the conventional definition commonly used in the signage industry has a unit expressed as feet/inch for viewing distance over character height. In addition, legible characters presented in the built environments subtend solid angles in a wide range from $1.783 \times 10^{-13} \mathrm{sr}$ for normal eyesight level $20 / 20$ of population, which is recommended as the minimum value for enhanced legible environments, to probably a maximum $2 \pi$ sr subtended by the half viewing sphere in front of the observer. Thus, the value of new Legibility Index approximately ranges from 0.4 to $2.373 \times 10^{6}$ (legible for acuity $20 / 20$ ), or larger (for acuity better than $20 / 20$ ), no unit.

\section{CONCLUSIONS}

Capturing how people recognize text and graphics, a new definition of the Legibility Index $\left(L I^{\prime}\right)$ based on the three-dimensional solid angle $\omega$ has been developed in this research, as $L I^{\prime}=\sqrt{1 / \omega}$. After substituting the definition of solid angle $\omega$, this new definition can be further expressed as $L I^{\prime}=(D / \sqrt{A})(\cos$ $\xi)^{-0.5}$ ( $D$ is the legibility distance; $A$ is the normal character area; $\xi$ is the incident angle). For text and graphics using width and height to define their normal area, $L I^{\prime}=(D \cdot \sqrt{r} / H)(\cos \phi)^{-0.5}(\cos \alpha)^{-0.5}(H$ is the character height; $r$ is the height-to-width ratio; $\phi$ and $\alpha$ are the horizontal and vertical viewing angles). This equation has offered researchers a new measure for calculating the Legibility Index of text or graphics viewed not perpendicularly. It can also examine text and graphics viewed perpendicularly, by setting the incident angle to zero: $L I_{\text {perpendicular }}^{\prime}=(D / H) \cdot \sqrt{r}$. On the other hand, since character size $(H$ or $A$ ) is often desired in practice, this definition can also be used to predict the geometries and orientations of text or graphics viewed not perpendicularly, when legibility levels (measured using the Legibility Index) are assigned, using $H=(D$ $\left.\cdot \sqrt{r} / L I^{\prime}\right)(\cos \phi)^{-0.5}(\cos \alpha)^{-0.5}$, or $A=D^{2} /\left(\left(L I^{\prime}\right)^{2} \cdot \cos \phi \cos \alpha\right)$.

\section{ACKNOWLEDGMENTS}

This research has been facilitated by the University of Michigan Transportation Research Institute (UMTRI). We would like to thank Dr. Michael Flannagan and Mr. Brandon Schoettle in UMTRI's Human Factors Division for their help in providing equipment and other laboratory supplies. We also thank the anonymous reviewer for his/her comments on literature review on specifying visual stimuli.

\section{REFERENCES}

A preliminary version of this paper was delivered at the IESNA annual conference 2007.

Bailey I, Clear R, Berman S. 1993. Size as a determinant of reading speed. J Illum Eng Soc. 22(2):102-117.

Blackwell HR. 1946. Contrast thresholds of the human eye. J Opt Soc Am. 36(1 1):624-643.

Blackwell HR. 1959. Development and use of a quantitative method for specification of interior illumination levels on the basis of performance data. Illum Eng. 54(6):317-353.

Blackwell HR. 1970. Development of procedures and instruments for visual task evaluation. Illum Eng. 65(4):267-291. 
Blackwell HR. 1980. Description of a comprehensive family of relative contrast sensitivity (RCS) functions of luminance to reflect differences in size of task detail, task eccentricity and observer age. Ohio State University, Columbus, Ohio: Illuminating Engineering Research Institute; Available in Compendium of technical data in support of CIE publication No. 19/2. Illuminating Engineering Research Institute, New York, N.Y.

Blackwell HR, Blackwell OM. 1980. Population data for 140 normal 20-30 year olds for use in assessing some effects of lighting upon visual performance. J Illum Eng Soc. 9(3):158174.

Blackwell OM, Blackwell HR. 1971. Visual performance data for 156 normal observers of various ages. J Illum Eng Soc. 1(1):3-13.

Boff KR, Kaufman L, Thomas JP, editors. 1986. Handbook of perception and human performance: vol. 1. Sensory processes and perception. New York, NY: Wiley. 1296 p.

Bouma H, Legein CP, Melotte HEM, Zabel L. 1982. Is large print easy to read? Oral reading rate and word recognition of elderly subjects. IPO Annual Progress Report. 17:84-90.

Campbell FW, Robson JG. 1968. Application of fourier analysis to the visibility of gratings. J Physiol. 197:551-566.

Clear R, Berman S. 1990. Speed, Accuracy and VL. J Illum Eng Soc. 19(2):124-131.

Dember WN. 1965. The psychology of perception. New York: Holt, Rinehart and Winson. $402 \mathrm{p}$.

Gove PB, Merriam-Webster editorial staff. 1986. Webster's Third New International Dictionary of the English Language Unabridged. Springfield, MA: Merriam-Webster.

Hendee WR, Wells PNT, editors. 1993. The perception of visual information. New York, NY: Springer-Verlag. 409 p.

Holick AJ, Carlson PJ. 2002. Model of overhead-sign luminance needed for legibility. Transp Res Rec. 1801:80-86.

Howell W, Kraft C. 1960. Size, blur and contrast as variables affecting the legibility of alphanumeric symbols on radar-type displays. OH: Wright- Patterson AFB. WADC Technical Report 59-536.

Kambich DG. 1991. An alternative relative visual performance model. J Illum. Eng. Soc. 20(1):19-27.

Kristofferson AB. 1957. Visual detection as influenced by target form in discrimination a related to military problems. Washington DC: National Academy of Sciences. National research council publication 561:p109-127.

Legge GE, Pelli DG, Rubin GS, Schleske MM. 1985. Psychophysics of reading: I normal vision. Vision Res. 25(2):239-252.

Moon P, Spencer D. 1944. Visual data applied to lighting design. J Opt Soc Am. 34(10):605617 .

Rea MS. 1986. Toward a model of visual performance: foundations and data. J Illum. Eng. Soc. 15(2):41-57.

Rea MS, editor. 2000. The IESNA lighting handbook: reference and application. $9^{\text {th }}$ ed. New York, NY: The Illuminating Engineering Society of North America. Lighting calculation; p 9-1 $-9-72$.

Rea MS, Quellette MJ. 1988. Visual performance using reaction times. Light Res Tech. 20(4): $139-153$.

Rea MS, Quellette MJ. 1991. Relative visual performance: a basis for application. Light Res Tech. 23(3):135-144.

Rubin GS, Siegel KA. 1984. Recognition of low-pass filtered letters and faces. Paper presented at annual meeting of Association for Research in Vision and Ophthalmology, Sarasota, FL. 
Shlaer S. 1937. The relation between visual acuity and illumination. J Gen Physiol. 21:165188.

Shlaer S, Smith E, Chase A. 1942. Visual acuity and illumination in different spectral regions. J Gen Physiol. 25(4):553-569.

United States Sign Council. 1998. Sign legibility, overview and calculation methodology, sign legibility index. Member Resource Folio/Legislative Information. Bristol, PA: United States Sign Council. 2 p.

Wandell BA. 1995. Foundation of vision. Sunderland, MA: Sinauer Associates. 476 p.

Wolken JJ. 1966. Vision, biophysics and biochemistry of the retinal photoreceptors. Springfield, IL: Charles C Thomas. 193 p.

Zwahlen HT, Schnell T. 1995. A combined age-background luminance contrast multiplication function to adjust the human contrast threshold more accurately in visibility and legibility. Proceedings of Progress in Automobile Lighting, 240-247. Athens, OH: Ohio University.

Zwahlen HT, Schnell T. 1999. Legibility of traffic sign text and symbols. Trans Res Rec. 1692:142-151. 